\title{
Analysis of Air Bubble Deformation Subjected to Uniform Electric Field in Liquid Dielectric
}

\author{
M. Talaat", A. El-Zein \\ Electrical Power \& Machines Department, Faculty of Engineering, Zagazig University, Zagazig, Postcode 44519, Egypt
}

\begin{abstract}
A new numerical analysis has been carried out in order to determine the electrostatic pressure acting on the liquid-air interface. Also by aid of photographic recording the surface tension at the equator and poles is determined, the internal pressure is calculated and the relationship with the voltage square is enlightened. The analysis of the unstable bubble ejected or compressed against the sphere electrode with sharp edge protrusion, is explained and the values of charge under an applied electrical field at ejection condition are given. The breakdown electric field of the transformer oil is calculated, and a new breakdown voltage equation linking the compressed conducting bubble to the breakdown field is proposed. A new ratio of calculated electric charge at ejection mode relative to the value of ejected electric field is achieved. Also the breakdown voltage can be estimated by knowing the value of the voltage at compressed mode, using a new addressed space charge formation factor which is equal to 1.1. The results have been assessed through comparison with available analytical and experimental data.
\end{abstract}

Keywords Deformed Air Bubble, Uniform Electric Field, Dielectric Liquid, Breakdown in Liquid Dielectric, Sphere to Plane Gap, Space Charge Calculation, Streamer Formation

\section{Introduction}

Liquid dielectric improvement is a very interesting subject for the development of higher voltage levels of transmission system and corresponding components such as transformers, circuit breakers, capacitors, etc. Also, the required "rep-rate" switch using high pressure dielectric liquids is essential for future directed energy applications[1]. Thus, the pressure analysis is a very important way for determining the condition of our liquids. The interface problem is also an important item to study the pressure stability analysis[2]. Therefore, the study of air bubbles existing in dielectric liquid medium, and their effect on the breakdown characteristics were carried out by many authors[3-14]. Also, the stressed liquid volume and stressed electrode area for cryogenic liquids were reported by[15]. These authors showed that the breakdown increased with larger stressed liquid volume, which means, minimum air voids size. And the area effect under the existence of micro-protrusion on the electrode surface dominated the breakdown strength for electrodes with small stressed liquid volume or rough surface [15].

The induced electric pressure characteristics under applied voltage are similar to that of current[16,17]. The effect of additives to dielectric liquids plays an important role in its

* Corresponding author:

m_mtalaat@zu.edu.eg (M. Talaat)

Published online at http://journal.sapub.org/ijea

Copyright (C 2012 Scientific \& Academic Publishing. All Rights Reserved characteristics. For example the current and induced pressure can be raised linearly by the increase of moisture content till 5 and 3.2 respectively[17].

The pressure has been taken to support the theory, that liquid dielectric breakdown is started by the formation of gas bubbles in the liquid medium. Then, the bubble breakdown is considered the inception event leading to liquid breakdown[18]. Also, transformer oil with pressure up to $2.4 \mathrm{MPa}$ showed a breakdown strength gain of up to $35 \%-40 \%$, increase above the atmospheric pressure[19].

In this work, the role of bubbles in the streamer generation in liquid dielectrics subjected to an electric field is studied. It is intended to use the deformation of an air bubble located in a non-uniform electric field with different spaces between electrodes and different high voltage electrode diameter to study the pre-breakdown behavior and to explore which theory could explain our experimental results.

\section{Experimental Techniques}

Figure 1, shows the technique used for experimental recording $[20,21]$. The test cell consists of a transparent glass container of $5 \times 5 \times 20 \mathrm{~cm}$. The upper Perspex cover of the cell supports the inlet copper pipe of $6 \mathrm{~mm}$ with inner diameter of $3 \mathrm{~mm}$; this pipe is screwed externally to $5 \mathrm{~mm}$ from one side. The cover also supports an inlet, for dielectric liquid input. The pipe carries a high voltage sphere termination which is open till the sphere tip and screwed internally to $5 \mathrm{~mm}$ to be erected in the mentioned copper pipe. The other 
side of the pipe is connected to the injection air bubble system. Also the bottom of the test cell is made of Perspex and supports the grounded plane, $3 \mathrm{~cm}$ in diameter. The grounded plane position can be changed to adjust the tested gap against high voltage sphere electrode. In the present experiment one sphere termination of $11.28 \mathrm{~mm}$ diameter has been used.

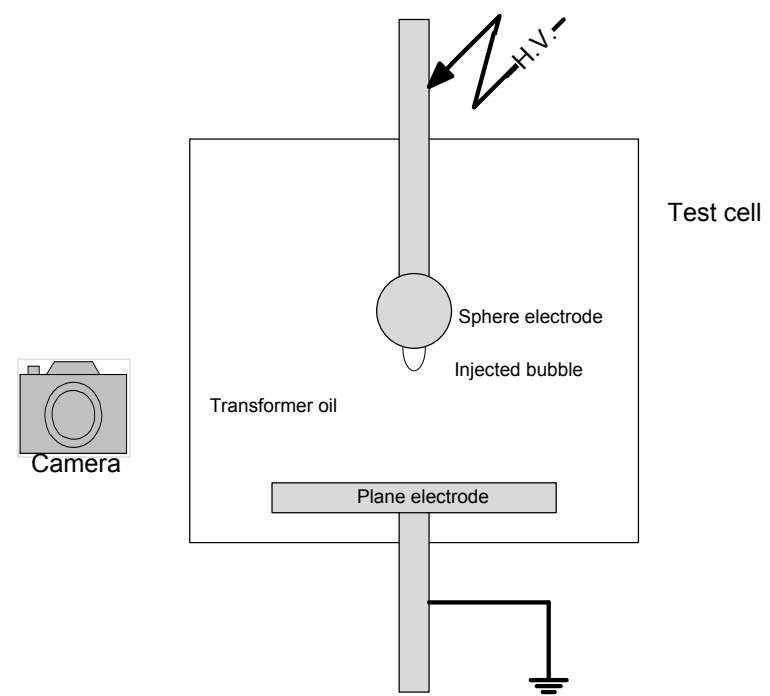

Figure 1. Scheme of a bubble immersed in a liquid dielectric under a sphere-to-plane configuration

The tests were carried out using $0 \sim 60 \mathrm{kV}$ D.C. power supply with negative polarity. The high voltage D.C. supply was connected to the sphere electrode, using $0.5 \mathrm{M} \Omega$ series resistor.

\subsection{Experimental Procedures}

1. The high voltage electrode was terminated with the required sphere electrode. The grounded plane was adjusted to the required distance by using the special screwing rod, which passed through the lower termination. After that, the glass test cell container was filled to a required hydrostatic head above sphere tip. The test gap was adjusted to required distance.

2. The injection system was adjusted to initial condition by the aid of coarse syringe adjustment, and stopcocks system containing valves. After that the air bubble was injected by using micrometer loaded syringe.

3. The high voltage D.C starts to rise gradually with $2 \mathrm{kV}$ step. At each voltage step the air bubble shape was photographed.

4. This photograph recording was performed by increasing the applied voltage until the air bubble compressed or ejected from the sphere tip.

\subsection{Experimental Calculation}

1. The internal pressure of the bubble can be calculated by knowing the minor and major semi-axis from photographic recording using Krasucki equations for the pressure at the poles, and at the equator [6].

At poles,

$$
P_{i}=P_{e p}+\frac{2 \sigma \gamma}{b}+P_{h e}
$$

At equator,

$$
P_{i}=P_{e q}+\frac{\sigma\left(\gamma^{2}+1\right)}{b \gamma^{2}}+P_{e}
$$

where, $P_{i}$ is the internal pressure of the bubble, $P_{e p}$ is the electrical pressure at the pole, $P_{e q}$ is the electrical pressure at the equator, $P_{h}$ is the hydrostatic pressure above the bubble, $\sigma$ is the surface tension of the dielectric medium, $\gamma$ is the ratio of the bubble elongation and $b$ is the minor semi-axis.

2 . The cross section area of the bubble can be also calculated from the photographic recording by the aid of ellipse area equation.

$$
\text { Area }=\pi \times a \times b
$$

where, $a$ is the major semi-axis and $b$ is the minor semi-axis

\section{Method of Analysis}

\subsection{Deformed Air Bubble}

\subsubsection{Stable Air Bubble}

The stable air bubble case is taken in the low voltage region, where the relation between the voltage increasing and elongation of air bubble is in the linear region.

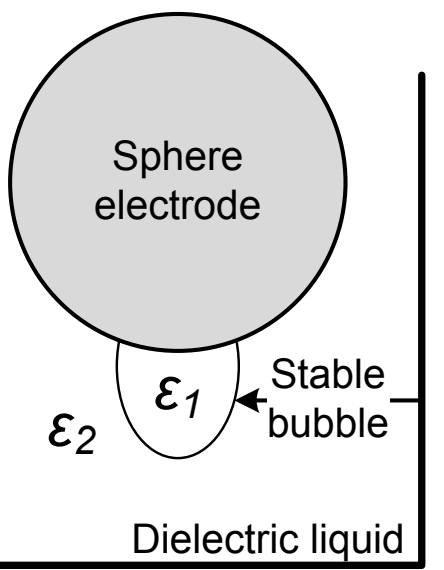

Figure 2. Table bubble immersed in a dielectric medium

The average electrostatic pressure $\left(P_{e}\right)$ is used as an effective electrostatic traction acting on the boundary of a spherical bubble [14]. This pressure given as:

$$
P_{e}=\frac{3}{2} \cdot \frac{\varepsilon_{2}-\varepsilon_{1}}{2 \varepsilon_{2}+\varepsilon_{1}} \cdot \varepsilon_{2} \varepsilon_{o} E^{2}
$$

where, $\varepsilon_{1}$ and $\varepsilon_{2}$ are the relative permittivity of the air and dielectric liquid respectively, i.e., $\varepsilon_{2}>\varepsilon_{1}$.

For stable air bubble, $P_{e}$, tends to press the bubble against the sphere electrode.

\subsubsection{Unstable air bubble}

For unstable bubble, the accumulated charge on the air-liquid interface increases. 


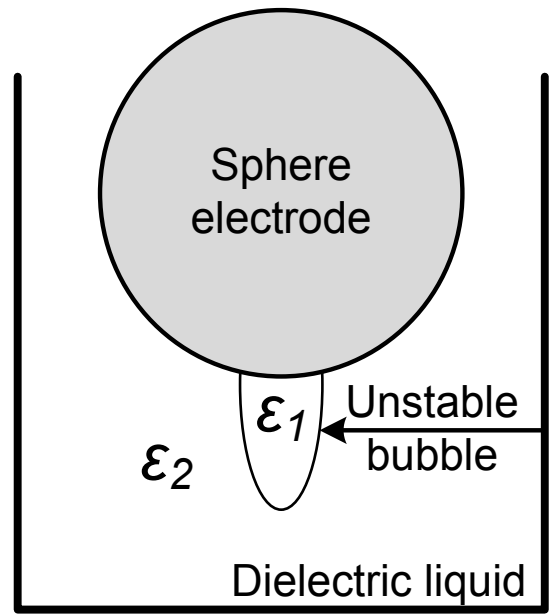

Figure 3. Unstable bubble immersed in a dielectric medium

This charge, which has the same sign of the spherical electrode, increases the Columbic pressure $\left(P_{c}\right)$

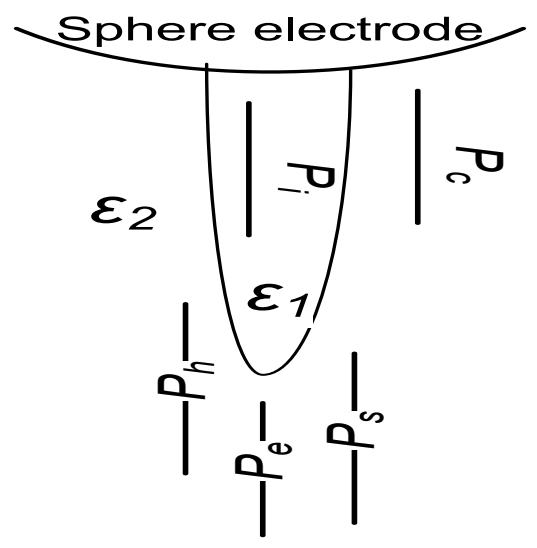

Figure 4. Pressure analysis for unstable bubble

For more bubble analysis, we must notice the following for air bubble balanced pressures.

$$
P_{e}+P_{h}+P_{s}=P_{i}+P_{c}
$$

where, $P_{h}, P_{s}$ and $P_{i}$ are hydrostatic pressure, surface tension pressure and internal air bubble pressure respectively.

By increasing the applied voltage, $P_{c}$ increases also, and if we assumed that $P_{h}+P_{s}=P_{i}$ which is correct at the instant of zero applied voltage. Therefore, $P_{c} \leq P_{e}$ for unstable bubble which will be compressed or eject according to relative value of $P_{c}$ with respect to $P_{e}$ i.e., three modes arises:

- First mode: $P_{e}>P_{c}$ the bubble compressed.

- Second mode: $P_{e}<P_{c}$ the bubble ejected.

- Third mode: $P_{e}=P_{c}$ the bubble becomes unstable.

which can be considered as a new finding for declaration of what happen to injected air bubble under different experimental high voltage conditions.

\subsection{Space Charge Calculation}

If the space charge is $(q)$ then the force on this charge due to applied electric field $(E)$ will be,

$$
F=q E
$$

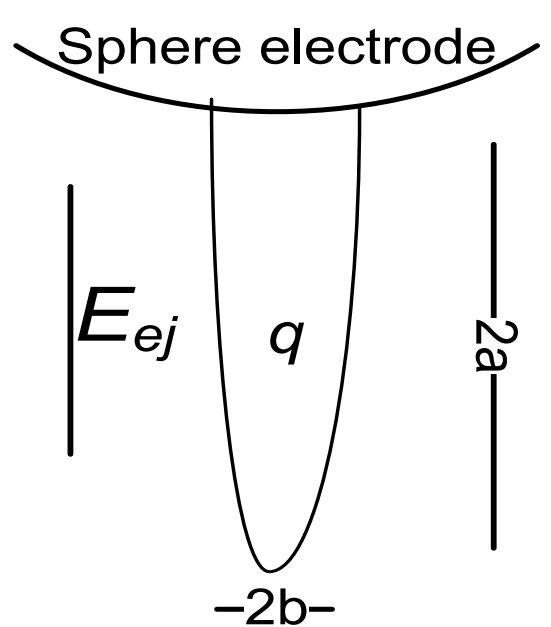

Figure 5. Unstable bubble in a dielectric medium for ejection case

This force can be called the Columbic force, which can be given at the equator as follows:

$$
F_{c}=P_{c} \times \text { area }
$$

If the bubble is unstable, $P_{e}=P_{c}$, the following equation can be given:

$$
\frac{1}{\pi b^{2}} q E_{e j}=\frac{3}{2} \cdot \frac{\varepsilon_{2}-\varepsilon_{1}}{2 \varepsilon_{2}+\varepsilon_{1}} \cdot \varepsilon_{2} \varepsilon_{o} E_{e j}^{2}
$$

where, $\pi b^{2}$ is the elliptical elongated bubble cross section area and $E_{e j}$ is the value of the applied electric field at instance of bubble ejection.

$$
q=\frac{3}{2} \cdot \frac{\varepsilon_{2}-\varepsilon_{1}}{2 \varepsilon_{2}+\varepsilon_{1}} \cdot \varepsilon_{2} \varepsilon_{o} E_{e j} \pi b^{2}
$$

where, $\varepsilon_{1}=1$ for air bubble and $\varepsilon_{2}=2.1$ for transformer oil. The space charge under the case of transformer oil,

$$
q=5.899 \times 10^{-12}\left(\pi b^{2}\right) E_{e j}
$$

\subsection{Breakdown Analysis}

If the unstable bubble was not ejected, with further increasing of the applied voltage, the ionization inside bubble increases also. At higher voltages this bubble will be converted to conducting one, and compressed against the sphere electrode with sharp end protrusion.

The electric field intensity ahead this protrusion tip will act to push off the liquid from this tip. At balance condition this electrical induced pressure must overcome the hydrostatic and atmospheric pressures.

When the bubble is converted from dielectric to conducting medium the electric pressure $P_{e}$ could be given according to equation,

$$
P_{e}=-\frac{3}{2} \cdot \varepsilon_{2} \varepsilon_{o} E_{t}^{2}
$$

where, $E_{t}$ is the electric field at the tip of the compressed air bubble i.e., conducting medium.

At this condition,

$$
P_{e}=P_{h}+P_{a}
$$

where, $P_{a}$ is the atmospheric pressure which equal $10^{5}$ $\mathrm{N} / \mathrm{m}^{2}$ 


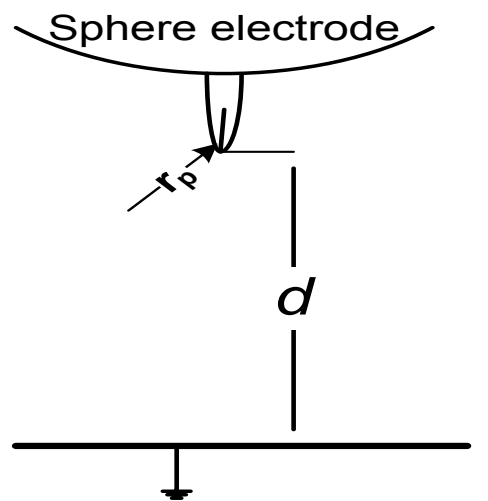

Figure 6. Compressed bubble in a dielectric for breakdown case

The applied voltage required to produce this value of field can be calculated from the following equation:

$$
E_{t}=\frac{2 V}{r_{P} \ln \left(4 d / r_{p}\right)}
$$

where, $r_{p}$ is the radius of the compressed air bubble tip, $d$ is the distance from bubble tip to plane electrode and $V$ is the applied voltage.

\subsection{Compressed Air Bubble Tip Radius Estimation}

The tip radius of the compressed air bubble can be measured from the photographic recording analysis as following:

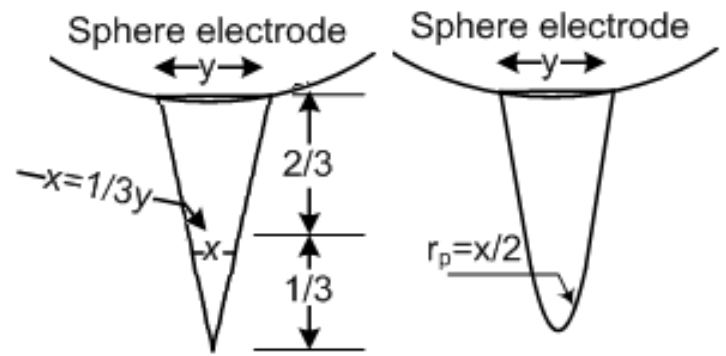

Figure 7. Schematic figure for radius calculation of compressed air bubble

We can consider the protrusion tip as a cone with base " $y$ " as shown, at $2 / 3$ the height of the cone we can consider the distance " $x "$ as the diameter of the tip as shown in the schematic figure. From this Figure $r_{p}=x / 2$ and $y=3 x=6 r_{p}$.

Accordingly the voltage at which the gap breaks down can be calculated from equation (13) by the measured experimental values of $y$ as follows:

$$
V_{b}=\frac{y}{12} \ln \left(\frac{4 d \times 6}{y}\right) E_{t}
$$

where, $V_{b}$ is the breakdown voltage.

\section{Results and Discussion}

\subsection{Experimental Results}

Sphere electrodes of $10,11.28,13.22,14.33$ and $15.8 \mathrm{~mm}$ diameter with a hydrostatic pressure of $4 \mathrm{~cm}$ oil were used. The test has been carried out to detect the behavior of air bubble under ascending mode against applied voltage, for 3 , 4, 5, 6, 7, 8 and $13 \mathrm{~mm}$ gap distances.
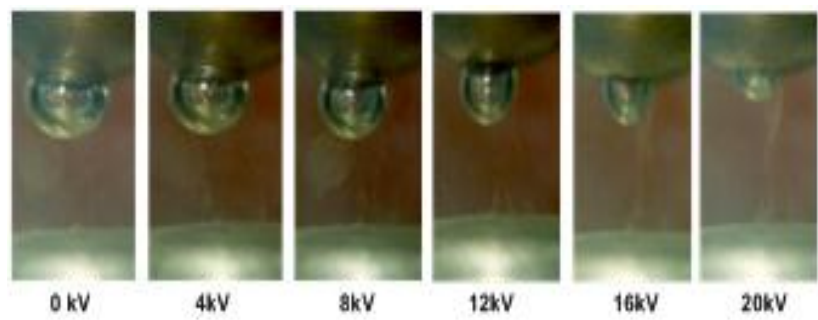

$16 k \mathrm{~V}$

$20 \mathrm{kV}$

Figure 8. Deformation process of air bubble for gap $6 \mathrm{~mm}$ sphere $11.28 \mathrm{~mm}$

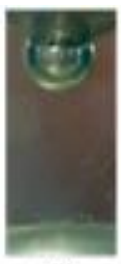

OKV

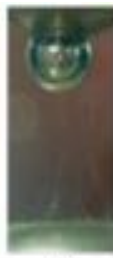

$4 \mathrm{KV}$

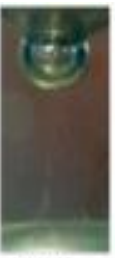

BKV

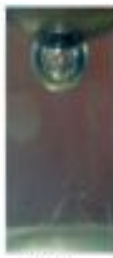

$12 \mathrm{KV}$

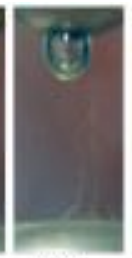

$16 \mathrm{kV}$

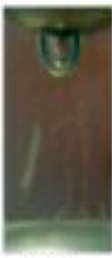

20kV

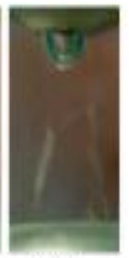

$24 k V$
Figure 9. Deformation process of air bubble for gap $8 \mathrm{~mm}$ sphere $11.28 \mathrm{~mm}$
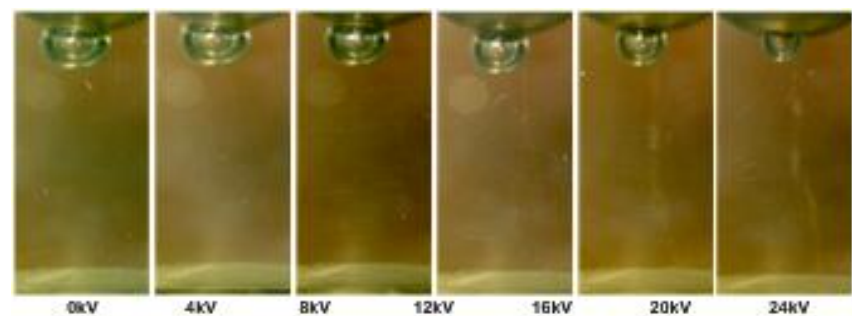

Figure 10. Deformation process of air bubble for gap $14 \mathrm{~mm}$ sphere $11.28 \mathrm{~mm}$

From Figures 8, 9 and 10, it is clear that the bubble assumes nearly a spheroid shape at low applied voltage and then it starts to elongate assuming an elliptical shape. With further increases of the applied voltage the bubble enter the sphere tip electrode, Figure 10, or eject from the gap, Figure 11 , or becomes unstable bubble, Figure 12.

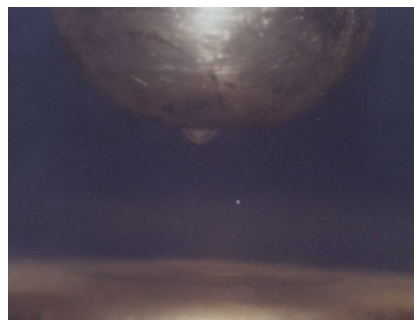

Figure 11. Compressed air bubble for gap $6 \mathrm{~mm}$ sphere $15.8 \mathrm{~mm}$

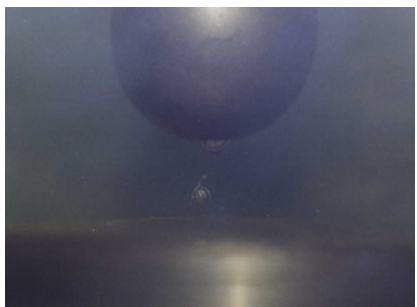

Figure 12. Ejected air bubble for gap $4 \mathrm{~mm}$ sphere $10 \mathrm{~mm}$ 


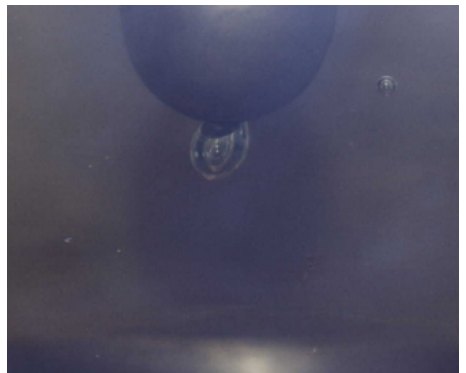

Figure 13. Unstable air bubble for gap $8 \mathrm{~mm}$ sphere $10 \mathrm{~mm}$

\subsection{Space Charge Calculation}

In order to demonstrate the proposed approach, Table 1 described the ejection or unstable bubble data obtained from experimental, these data required for calculating the space charge using equation (10).

Table 1. Voltage values in case of (ejected or unstable bubble modes) for different gap spacing using various sphere diameters

\begin{tabular}{|c|c|c|c|c|}
\hline \multirow{2}{*}{$\begin{array}{c}\text { Gap } \\
\text { distant } \\
(\mathrm{mm})\end{array}$} & \multicolumn{4}{|c|}{ Sphere diameter $(\mathrm{mm})$} \\
\hline & 10 & 13.22 & 14.33 & 15.8 \\
\hline 7 & & $15 \mathrm{kV}$ unstable & & \\
\hline 8 & & $17 \mathrm{kV}$ unstable & & $20 \mathrm{kV}$ eject \\
\hline 9 & & $18 \mathrm{kV}$ unstable & $18 \mathrm{kV}$ eject & \\
\hline 10 & & $18 \mathrm{kV}$ eject & $18 \mathrm{kV}$ eject & \\
\hline 11 & & $19 \mathrm{kV}$ eject & $20 \mathrm{kV}$ unstable & \\
\hline 12 & & $21 \mathrm{kV}$ unstable & $22 \mathrm{kV}$ unstable & \\
\hline 13 & $\begin{array}{r}20 \mathrm{kV} \\
\text { eject }\end{array}$ & $23 \mathrm{kV}$ unstable & $23 \mathrm{kV}$ unstable & \\
\hline 14 & $\begin{array}{r}28 \mathrm{kV} \\
\text { eject }\end{array}$ & $24 \mathrm{kV}$ unstable & & $34 \mathrm{kV}$ eject \\
\hline
\end{tabular}

The values of the space charge is calculated for transformer oil using equation (10) for different sphere terminations and tabulated in Table 2.

From Table 2, the required charge value $(q)$ for bubble ejection related to ejection electric field $\left(q / E_{e j}\right)$ is nearly equals to $22.4 \times 10^{-13} \mathrm{C} . \mathrm{cm} / \mathrm{k} V$.

Table 2. Space charge calculation

\begin{tabular}{|c|c|c|c|c|c|c|}
\hline \multirow[b]{2}{*}{$\begin{array}{c}\text { Sphere } \\
\text { diame- } \\
\text { ter } \\
(\mathrm{mm})\end{array}$} & \multicolumn{4}{|c|}{ Parameters required for equation (10) } & \multirow{2}{*}{$\begin{array}{c}\text { Space } \\
\text { charge } \\
\times \\
10^{-11} \\
(\mathrm{C})\end{array}$} & \multirow[b]{2}{*}{$\begin{array}{c}\mathrm{q} / \mathrm{E}_{\mathrm{ej}} \\
\times 10^{-13} \\
\text { C.cm/k } \\
\quad \mathrm{V}\end{array}$} \\
\hline & $\begin{array}{c}\text { Gap } \\
\text { distant } \\
(\mathrm{mm})\end{array}$ & $\begin{array}{c}\text { Ejec- } \\
\text { tion } \\
\text { voltage } \\
(\mathrm{kV})\end{array}$ & $\begin{array}{c}\mathrm{E}_{\mathrm{ej}} \\
(\mathrm{kV} / \mathrm{c} \\
\mathrm{m})\end{array}$ & $\begin{array}{c}\mathrm{b} \\
(\mathrm{mm})\end{array}$ & & \\
\hline 10 & 13 & 20 & 25 & 1.1 & 5.6 & 22.416 \\
\hline 10 & 14 & 28 & 34.45 & 1.1 & 7.725 & 22.42 \\
\hline 15.8 & 8 & 20 & 22.396 & 1.2 & 5.976 & 26.686 \\
\hline 15.8 & 14 & 34 & 30.04 & 1.1 & 6.736 & 22.424 \\
\hline
\end{tabular}

\subsection{Electric Field Calculation}

\subsubsection{Streamer Formation}

For streamer formation the number of electrons per avalanche, ne, needed are $108<n_{e}<109$ as given by[22].

From the previous values of $\mathrm{q}$ at ejection condition the number of equivalent electrons ranges between $3.735 \times 108$ at gap $8 \mathrm{~mm}$ and $4.21 \times 108$ at gap $14 \mathrm{~mm}$, for sphere 15.8 $\mathrm{mm}$ and $3.5 \times 108$ at gap $13 \mathrm{~mm}$, and $4.83 \times 108$ at gap $14 \mathrm{~mm}$, for sphere $10 \mathrm{~mm}$.

Therefore if we consider the number of electrons per avalanche equal to $10^{9}$ electrons for streamer formation, then the charge will equal to $q=e \times 10^{9}$, where $e$ is the electron charge value, $e=1.6 \times 10^{-19} \mathrm{C}$, then $q=1.6 \times 10^{-10} \mathrm{C}$.

Under our condition this charge may be achieved at fields equal to $59.9 \mathrm{kV} / \mathrm{cm}$ and $71.35 \mathrm{kV} / \mathrm{cm}$ for gap distance of 8 and $14 \mathrm{~mm}$ respectively sphere $15.8 \mathrm{~mm}$. Also this field equal to $71.35 \mathrm{kV} / \mathrm{cm}$ for gap distances of 13 and $14 \mathrm{~mm}$ sphere $10 \mathrm{~mm}$.

\subsubsection{Breakdown Analysis}

When the bubble is converted from dielectric to conducting medium the electric pressure $P_{e}$ could be given according to equation (12), using $h=40 \mathrm{~mm} .0 \mathrm{il}, g=9.81 \mathrm{~m} / \mathrm{s}^{2}$, $\rho=880 \mathrm{~kg} / \mathrm{m}^{3}, \varepsilon_{2}=2.1$ for transformer oil to determine the value of $P_{h}=\rho g h=345.3 \mathrm{~N} / \mathrm{m}^{2}$, from equation (12) the value of $P_{e}=10^{5} \mathrm{~N} / \mathrm{m}^{2}$, using this value in equation (11), then

$$
\frac{3}{2} \cdot \varepsilon_{2} \varepsilon_{o} E_{t}^{2}=10^{5}
$$

$E_{t}=598.8 \mathrm{kV} / \mathrm{cm}$, which is in accordance with that given to be $570 \mathrm{kV} / \mathrm{cm}$ by[23-25].

The voltage at which the gap breaks down can be calculated from equation (14).

Table 3 shows the experimental measured voltage at compressed bubble condition, $V_{c}$, and the breakdown voltage $V_{b}$ given by equation (14).

From this Table it is clear that the average value of the ratio $V_{b} / V_{c}$ is higher than one, this value can be called a space charge formation factor $(m)$, which reaches a mean value of 1.1

Table 3. The experimental measured $V_{c}$ and the calculated $V_{b}$

\begin{tabular}{c|c|c|c|c|c|c}
\hline \multirow{2}{*}{$\begin{array}{c}\text { Gap } \\
\text { distant } \\
(\mathrm{mm})\end{array}$} & \multicolumn{6}{|c}{ Sphere diameter $(15.8 \mathrm{~mm})$} \\
\cline { 2 - 7 }$(\mathrm{kV})$ & $\begin{array}{c}\mathrm{y} \\
(\mathrm{mm})\end{array}$ & $\mathrm{x}(\mathrm{mm})$ & $\mathrm{r}_{\mathrm{p}}(\mathrm{mm})$ & $\mathrm{V}_{\mathrm{b}}(\mathrm{kV})$ & $\begin{array}{c}m \\
=\mathrm{V}_{\mathrm{b}} / \mathrm{V}_{\mathrm{c}}\end{array}$ \\
\hline 3 & 16 & 0.75 & 0.25 & 0.125 & 17.08 & 1.0675 \\
\hline 5 & 23 & 1 & 0.333 & 0.1666 & 23.89 & 1.038 \\
\hline 6 & 26 & 1 & 0.333 & 0.1666 & 24.79 & 0.95 \\
\hline 8 & 26 & 1.25 & 0.4166 & 0.208 & 31.4 & 1.2 \\
\hline 12 & 37 & 1.5 & 0.5 & 0.25 & 39.35 & 1.063 \\
\hline 14 & 42 & 1.75 & 0.583 & 0.2915 & 45.9 & 1.09 \\
\hline
\end{tabular}

\section{Conclusions}

In this paper, an effort was made to outline the different stages of pre-breakdown in dielectric liquid, "transformer oil", by using the deformation of an artificial air bubble injected at high voltage electrode between different gaps, and different high voltage electrodes diameters with constant hydrostatic head in a non-uniform electric field.

Based on the finding of the present work the following salient points may conclude this paper:

1. The injected air bubble under existing hydrostatic head 
and no applied voltage has a prolate spheroid shape, with the elongation of bubble in the horizontal axis.

2. As the voltage increase the internal pressure of the bubble also increase as approximately linear relation until a certain value for which the bubble compressed and the non-conducting bubble convert to a conducting one. After that, the spheroid shape assumes a conical shape. At this value, there is no air bubble but a conducting gas medium.

3. For unstable bubble, the accumulated charge on the air-liquid interface increases. This charge, which has the same sign of the spherical electrode, increases the coulombic pressure $\left(P_{c}\right)$. So that the analysis of the unstable bubble can be represented as: when the electrostatic pressure is higher than coulombic pressure the bubble compressed (first mode), and when it becomes smaller than it the bubble will be eject (second mode), also, when they are equal the bubble becomes unstable (third mode).

4. Under the analysis of unstable bubble, the calculated charge under applied electrical fields at ejection condition reveals that its ratio equals $22.424 \times 10^{-13} \mathrm{C} . \mathrm{cm} / \mathrm{kV}$.

5 . When the bubble not ejected and with further applied voltage increase, till it compressed against the sphere electrode, a sharp end protrusion appears. The estimated breakdown field intensity reaches a value of $598.8 \mathrm{kV} / \mathrm{cm}$.

6. A new equation of breakdown voltage inside the dielectric liquid has been deduced, by knowing the length of the compressed air bubble base.

7. The new space charge formation factor $(m)$, which approximately equals 1.1 , used to describe the compressed and breakdown voltage for unstable compressed bubble.

\section{REFERENCES}

[1] Joshuca J. leckbee, Randy D. Curry, Kenneth F. Mc Dorald, W. Ray Cravey, Glenn Anderson and Susan Heidyer "Design, Modeling, and Verification of a High - Pressure Liquid Dielectric Switch for Directed Energy Applications" IEEE Transactions on Plasma science Vol. 32, No.5, pp.1790-1798, 2004.

[2] F. Vega Reyes and A. Castellanos "The Electric Pressure Jump in the Hydraulic Model in a Non-Ohmic/Non-Ohmic Fluid Interface" IEEE CEIDP 2004, pp.118-121, 2004.

[3] A. Nosseir, A. El-Zein and E. Taha, "Experimental Determination of Electrically Induced Pressure in Dielectric Liquid under External Bubble Injection", International Symposium on Electrical Insulation, pp. 69 -72, 1986.

[4] A. Nosseir, I. F. Hashad, E. Taha and A. El-Zein, "Electrically Induced Pressure in Mirearal Oil under External Bubble Injection ", Journal of Electrostatics., Vol. 12, pp. 511-516, 1982.

[5] A. El-Zein, "Experimental Separation of Dielectroforetic Pressure in Insulating Liquids under Direct Positive Applied Voltages", 6th International Symposium on High Voltage Engineering, pp. 33.03, 1994.

[6] C. G. Garton and Z. Krasuski "Bubbles in Insulating Liquids:
Stability in an Electric Field" Proceedings of Royal Society of London. Series A, Vol. 280, pp. 211-226, 1964.

[7] A. El-Zein "Determine of Air Bubble Deformation and Surface Tension Pressure in Insulating Liquids under Electrical Applied Fields" Sixth International Symposium on High Voltage Engineering, USA 13.19, 1989.

[8] M. Sadeghzadeh-Araghi, M. I. Qureshi, W. G. Chadband, P. $\mathrm{K}$. Watson, "Measurement of the Growth of Cavities and EHD Instabilities During The Negative-Point Breakdown of Silicon Fluids" IEEE Transactions On Electrical Insulation, Vol. 26, No. 4, pp. $663-672,1991$.

[9] Y. C. Kweon, M. H. Kim, H. J. Cho and I. S. Kang "Study on the Deformation and Departure of a Bubble Attached to a Wall on DC/AC Electrical Fields" Int. J. Multiphase Flow, Vol. 24 No. 1 pp. 145-162, 1998.

[10] H. J. Cho, I. S. Kang, Y. C. Kweon and M. H. Kim "Numerical Study of The Behavior of Bubble Attached to a Tip in a Non uniform Electric Field" Int. J. Multiphase, Flow, Vol. 24, No. 3, pp. 479-498, 1998.

[11] Mohamed Chaker Zaghdoudia and Monique Lallemand "Study of the Behavior of a Bubble in an Electric Field: Steady Shape and Local Fluid Motion" Int. J. Therm. Sci. (2000) 39, 39-52, 2000.

[12] Brian K. Mori and W. Douglas Baines "Bubble Departure from Cavities" International Journal of Heat and Mass Transfer, Vol. 44, No. 4, pp. 771-783, 2001.

[13] M. Talaat "A Simulation Model of Fluid Flow and Streamlines Induced by Non-Uniform Electric Field" IEEE, 14th Inter. Middle East Power Systems Conference, pp. 371-375, Egypt, 2010.

[14] A. El-zein and M. Talaat, "A Numerical Model of Investigating the Electric Field in Dielectric Liquid" IEEE MELECON Conference 2010, pp. 393-397, 2010.

[15] N. Hayakawa, H. Sakakibara, H. Gashima, M. Hikita and H. Okubo "Breakdown Mechanism of Liquid Nitrogen Viewed from Area and Volume Effects" IEEE Trans. On Dielectrics and electrical Insulation, Vol. 4 No. 1, pp. 127-134, 1997.

[16] Otmar M. Stuetzer, "Ion Drag Pressure Generation" J. Appl. Phys., Vol. 30, Issue 7, pp. 984-994, 1959.

[17] Ichiro Kano, Ichiro Takashi and Tatsuo Nishina "Effects of Moisture Content in a Dielectric Liquid on Electrohydrodynamic Pumping" IEEE Transactions on Industry Applications, Vol. 45, No. 1, pp. 59-66, 2009.

[18] A. Sharbough, J. Devins and S. Razad, "Progress in The Field of Electric Breakdown in Dielectric Liquids" IEEE Trans. Elect. Insulation, Vol. 13, pp. 249-276, 1978.

[19] S. Oliver, R. Kattan and A. Denat "Numerical Study of Single-Vapor Bubble Dynamics in Insulating Liquids By Electrical Current Pulses" J. Appl. Phys, Vol. 71 No. 1 pp. 108-112, 1992.

[20] A. El-Zein and M. Talaat, "New Experimental Study of an Injected Air Bubble Deformation in Dielectric Liquid under Applied High D.C. Voltage Using Photographic Recording", IEEE 8th Inter. Middle East power systems conference, pp. 869-873, 2003.

[21] A. El-Zein and M. Talaat "Pre-Breakdown Analysis during 
the Deformation of An Artificial Air Bubble in Transformer Dielectric liquid Under High DC Negative Applied Voltage" IEEE 10th Inter. Middle East power systems conference, pp. 113-117, 2005.

[22] E. Aldea, P. Peeters, H. De Vries and M. C. M Van De Sanden, "Atmospheric Glow Stabilization. Do we Need Pre-Ionization?" Int. J. Surface and Coatings Technology, Vol. 200, Issues 1-4, pp. 46-50, 2005.

[23] K. Yoshino, "Dependence of Dielectric Breakdown of Liq- uids on Molecular Structure", IEEE Trans. Electr. Ins., Vol. EI-15, No. 3, pp. 186-200, June 1980.

[24] A. El-Zein "New Approach for Electric Breakdown Field Intensity Determination in Insulating Liquids on an Electrostatic Energy Stored Basis", IEEE Inter. Symp. Elect. Insu., pp. 422-425, 1994.

[25] N .Yu. Babaeva and G .V. Naidis, "Simulation of Positive Streamers in Liquid Argon and Xenon", IEEE 13th Inter. Conference on Dielectric Liquids, pp. 437-440, July 1999. 